\title{
Assessment of a smartphone-based monitoring system and its application
}

\author{
Hoyong Ahn*, Chuluong Choi* and Yeon $\mathrm{Yu}^{\star_{*}{ }_{+}}$ \\ *Department of Spatial Information Engineering, Pukyoung National University \\ **Spatial Information Institute, Pukyoung National University
}

\begin{abstract}
Information technology advances are allowing conventional surveillance systems to be combined with mobile communication technologies, creating ubiquitous monitoring systems. This paper proposes monitoring system that uses smart camera technology. We discuss the dependence of interior orientation parameters on calibration target sheets and compare the accuracy of a three-dimensional monitoring system with camera location calculated by space resectioning using a Digital Surface Model (DSM) generated from stereo images. A monitoring housing is designed to protect a camera from various weather conditions and to provide the camera for power generated from solar panel. A smart camera is installed in the monitoring housing. The smart camera is operated and controlled through an Android application. At last the accuracy of a three-dimensional monitoring system is evaluated using a DSM. The proposed system was then tested against a DSM created from ground control points determined by Global Positioning Systems (GPSs) and light detection and ranging data. The standard deviation of the differences between DSMs are less than $0.12 \mathrm{~m}$. Therefore the monitoring system is appropriate for extracting the information of objects' position and deformation as well as monitoring them. Through incorporation of components, such as camera housing, a solar power supply, the smart camera the system can be used as a ubiquitous monitoring system.
\end{abstract}

Key Words : Camera calibration, close-range photogrammetry, smart camera, ubiquitous monitoring

\section{Introduction}

Ubiquitous monitoring is the process of collecting and assimilating vast amounts of data from numerous electronic mobile sensory devices. The concept of ubiquitous monitoring was established as "the use of pervasive devices for the continuous collection of data in an Intelligent Pervasive Space (IPS) or other ubiquitous environments" by Moran (2010). Digital smart cameras are enabling precise and ubiquitous monitoring approaches with data forms, expanding traditional surveillance techniques (Botan, 2005; Singh, 2006 Coroama, 2004; Cas, 2005). Recently, unusual climate changes worldwide, including severe rain storms, earthquakes, volcanic eruptions, flooding, and landslides, have spurred the demand for environmental

Received June 20, 2014; Revised June 25, 2014; Accepted June 25, 2014.

†Corresponding Author: Yeon Yu (yeon.yeu@gmail.com)

This is an Open-Access article distributed under the terms of the Creative Commons Attribution Non-Commercial License (http://creativecommons. org/licenses/by-nc/3.0) which permits unrestricted non-commercial use, distribution, and reproduction in any medium, provided the original work is properly cited 
monitoring systems to prepare for these natural disasters, as well as to aid in recovery procedures. However, conventional monitoring systems require power supplies and communication lines, which are not cost effective, particularly for short-term observation.

The monitoring system used in this study is solarpowered and transfers data to a monitoring center via Wireless Fidelity (Wi-Fi) or 3GPP Long-Term Evolution (LTE) systems. The three-dimensional (3D) movement of the soil surface by detecting temporal changes through stereo photogrammetry, a process in which the projective geometry of an object into three dimensions is obtained from the photographic image and camera position. Using an approach similar to stereo photogrammetry, ubiquitous monitoring systems use the smart camera for application.

There are two types of cameras to consider, metric and non-metric, which differ according to their lens calibration. Metric cameras include lens calibration information for photogrammetric purposes, whereas, non-metric cameras do not. In the ubiquitous monitoring system proposed in this study, two nonmetric smart cameras were used. The 3-D spatial accuracy of the smart cameras was analyzed to investigate the feasibility of their use in the monitoring system. Specifically, 3-D (x, y, z) analysis calculated the accuracy of the Interior Orientation (IO) parameters. Additionally, the accuracy of various smartphone sensors was analyzed by comparing the Exterior Orientation (EO) parameters, position $(\mathrm{x}, \mathrm{y}, \mathrm{z})$ and altitude $(\omega, \phi, \kappa)$, using the Ground Control Points (GCP), with EO parameters provided by the Global Positioning System (GPS) (x, y, z), gyroscope ( $\omega, \phi$, $\kappa)$, accelerometer $(\omega, \phi, \kappa)$, and magnetometers $(\kappa)$, embedded in the smartphone (Pedersini, 1999). A Digital Surface Model (DSM) was generated using the $\mathrm{IO}$, Focal Length (FL), principal point (PP) coordinates, Charged-Coupled Device (CCD) pixel size, and the EO parameters calculated during the camera calibration procedure. A DSM was also created from light detection and ranging (LIDAR) data and smart camera/ smartphone camera imagery; this DSM was compared with the 3-D ( $\mathrm{x}, \mathrm{y}, \mathrm{z})$ DSM to evaluate the position accuracy of the proposed model.

\section{Methods and Materials}

Smart cameras, capable of image capture and processing have been used recently for object tracking and monitoring (Fleck, 2008; Tessens, 2013). In this study, we designed a ubiquitous monitoring system using android operated Smart Cameras (SCs) and smartphone cameras (SPs). The flowchart for this system is shown in Fig. 1, with the four steps outlined below:

Step 1: Camera calibration for lens distortion and the determination of the IO parameters using the PhotoModeler software (Eos Systems, Inc.). The SC and SPs used in this study were non-metric cameras, prone to lens distortion; thus, camera calibration was required to obtain good image quality.

Step 2: Data observation by Total Station, Geodetic GPS, and LiDAR. The GCPs were determined by ground surveying using Total Station after the base points were calculated using GPS. The point cloud data sets from terrestrial LiDAR, observed at several points, were geo-referenced.

Step 3: Validation of the 3-D monitoring system by comparison with the data using GCPs and LiDAR, as well as the IO parameters. This step was used to validate the accuracy of the target locations calculated from images taken by the calibrated camera. The camera IO, EO, and GCP parameters were required. The IO parameters of the camera correspond to the FL, PP coordinates, and pixel size calculated from Step 2. The EO parameters were the position $(\mathrm{x}, \mathrm{y}, \mathrm{z})$ and altitude $(\omega, \phi, \kappa)$ of the camera, provided by the smart camera sensors or GCPs. The resulting 3-D DSM created by the $\mathrm{IO}$ and $\mathrm{EO}$ values and stereo images was 


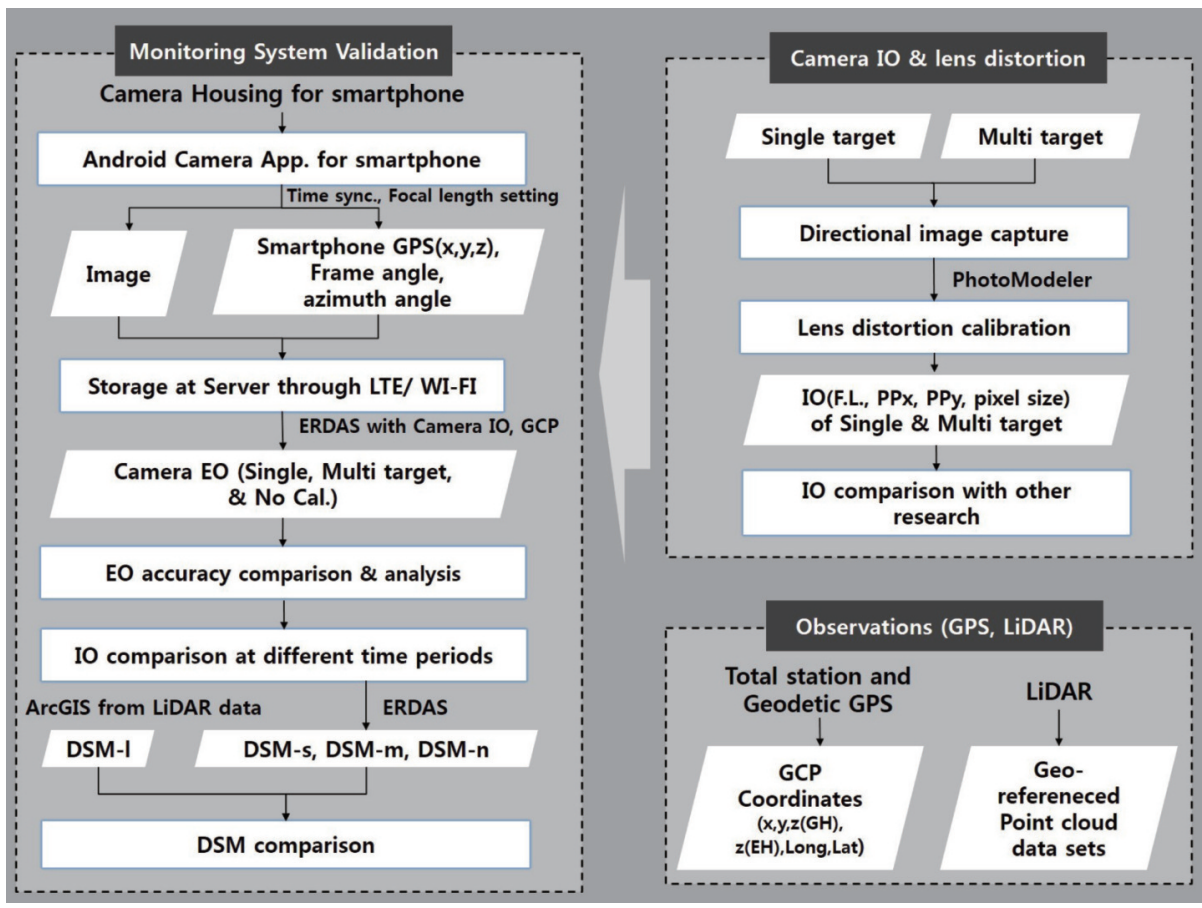

Fig. 1. Flowchart of involved in testing the newly developed monitoring system.

Table 1. Camera specifications

\begin{tabular}{|c|c|c|}
\hline & $\mathrm{SC}$ & SP \\
\hline & Type 2 & Type 1 \\
\hline Camera type & EK-KC120S & SHVE 250Ss \\
\hline Camera code NO. & & S5C73M3 \\
\hline Manufacturer & Samsung SEC & Samsung SEC \\
\hline CPU type & Quad core $1.4 \mathrm{GHz}$ & Quad core $1.6 \mathrm{GHz}$ \\
\hline OS type & Android 4.1.2(Jelly bean) & Android 4.1.2(Jelly bean) \\
\hline GPS type & GPS, GLONASS & GPS \\
\hline Memory & $\operatorname{In}(8 \mathrm{~Gb})$, Out(64Gb) & In(32Gb), Out(64Gb) \\
\hline Sensor type & BSI-CMOS & BSI-CMOS \\
\hline Sensor size & $1 / 2.3^{\prime \prime}$ & 1/2.3” \\
\hline CMOS Width, Height (mm) & $6.451,4.838$ & $4.569,3.427$ \\
\hline Image Width, Height (pixel) & 4608,3456 & 3264,2448 \\
\hline Focal Length by Company (mm) & $4.1 \sim 86.1$ & 3.7 \\
\hline Output format & \multicolumn{2}{|c|}{ JPEG } \\
\hline
\end{tabular}

Source: http://www.samsung.com/sec/

compared with the DSM created from LiDAR data (DSM-1).

\section{1) Equipment design}

Two cameras were used in this study: the SC (e.g., Samsung Galaxy Camera) and a SP (e.g., Samsung
Galaxy Note 2) (Table 1). Several studies have demonstrated the monitoring capabilities of the SP (Lee, 2012a; Kim, 2013a; Kim, 2013b).

The monitoring system consisted of the camera and its housing (i.e., the camera frame, external battery, leveling head, solar cell, and charger controller) ((a) 

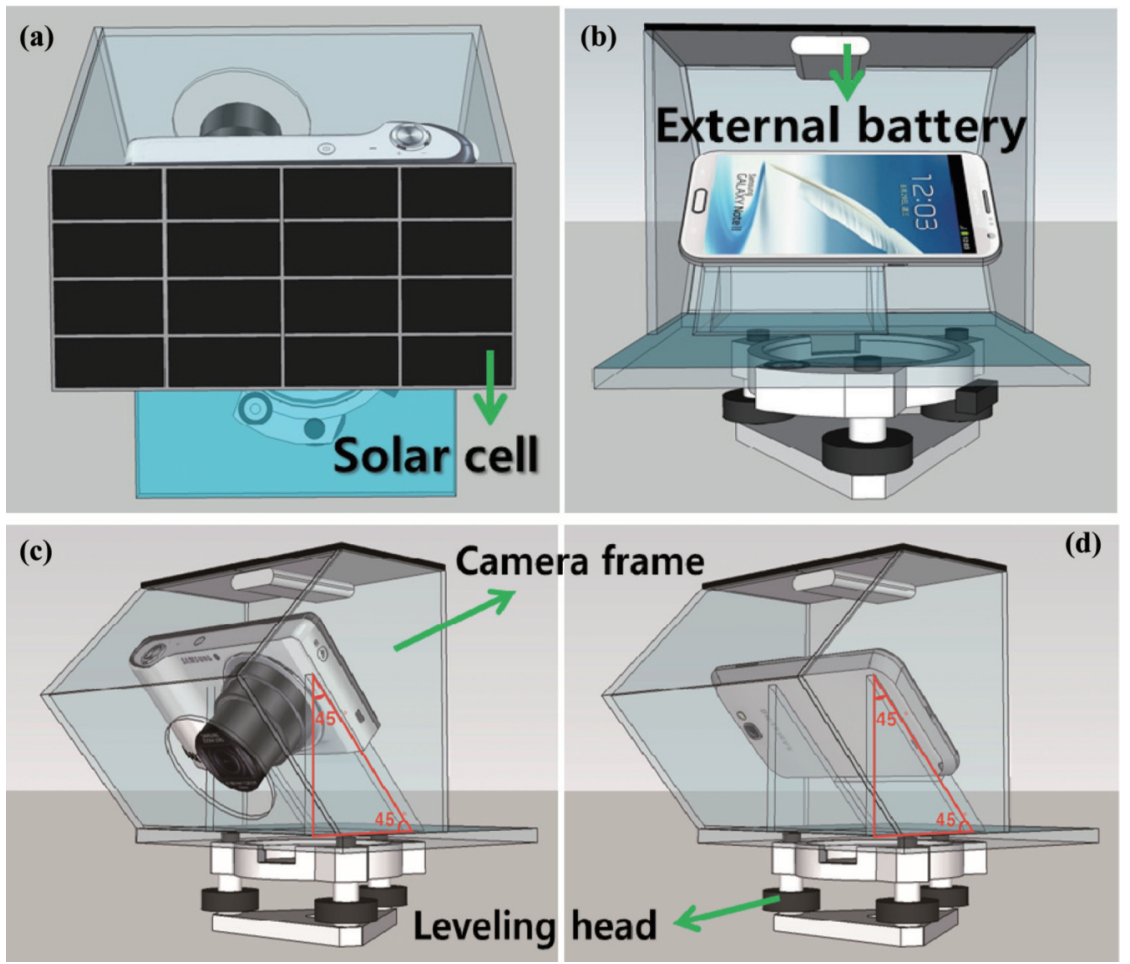

Fig. 2. Image acquisition system: (a) top view (b) rear view (c) and (d) 3-D view.

and (b)). The electricity generated by a 3-W solar cell was produced with 5 VDC 1 Ah through the charger controller, which was then stored in an external 14.8V, 3300-mAh lithium battery as 49 Watt, which enabled the camera system to last $\sim 1$ week without external power. A leveling head was used to keep the camera horizontal with respect to the ground. The acrylic camera housing was used to protect the camera from the external environment, such as rain, snow, and wind. The camera was aligned at a $45^{\circ}$ pitch angle by the camera support frame ((c) and (d)). As accelomenter or accelomenter built-in a smartphone is not enough for their accuracy to be used for EO parameters of camera (Joundi, 2011), it simplified EO parameters of camera using the supporting frame.

This study obtained stereo images using the SC and two sets of SPs. An Android application (app) was developed for effective processing of synchronization in taking the picture, equalizing the photo time intervals, and the number of images (Google 2012; Lee, 2012b; Kim, 2013b).

The main menu of this app consisted of four control buttons: monitoring, test capture, schedule, and setting. The monitoring button turned the monitoring system on or off. The test capture button allowed a check of the monitoring system operation. The schedule button assigned a start time for the monitoring. The setting button consisted of four tabs: monitoring, camera, saving, and sending. The monitoring tab set the synchronization of the camera timing and captured the time interval and the specified number of images. The camera tab enabled the image resolution, camera focus type (auto or infinite), and flash to be set. The saving tab provided an option for how the images were to be stored. The sending tab allowed camera images and image metadata to be sent via $\mathrm{Wi}-\mathrm{Fi}$ and/or LTE networks (Fig. 3). Image metadata included the time of image acquisition and the observation values measured by android-operated sensors such as the GPS, accelerometer, and gravimeter (Google, 2012). 

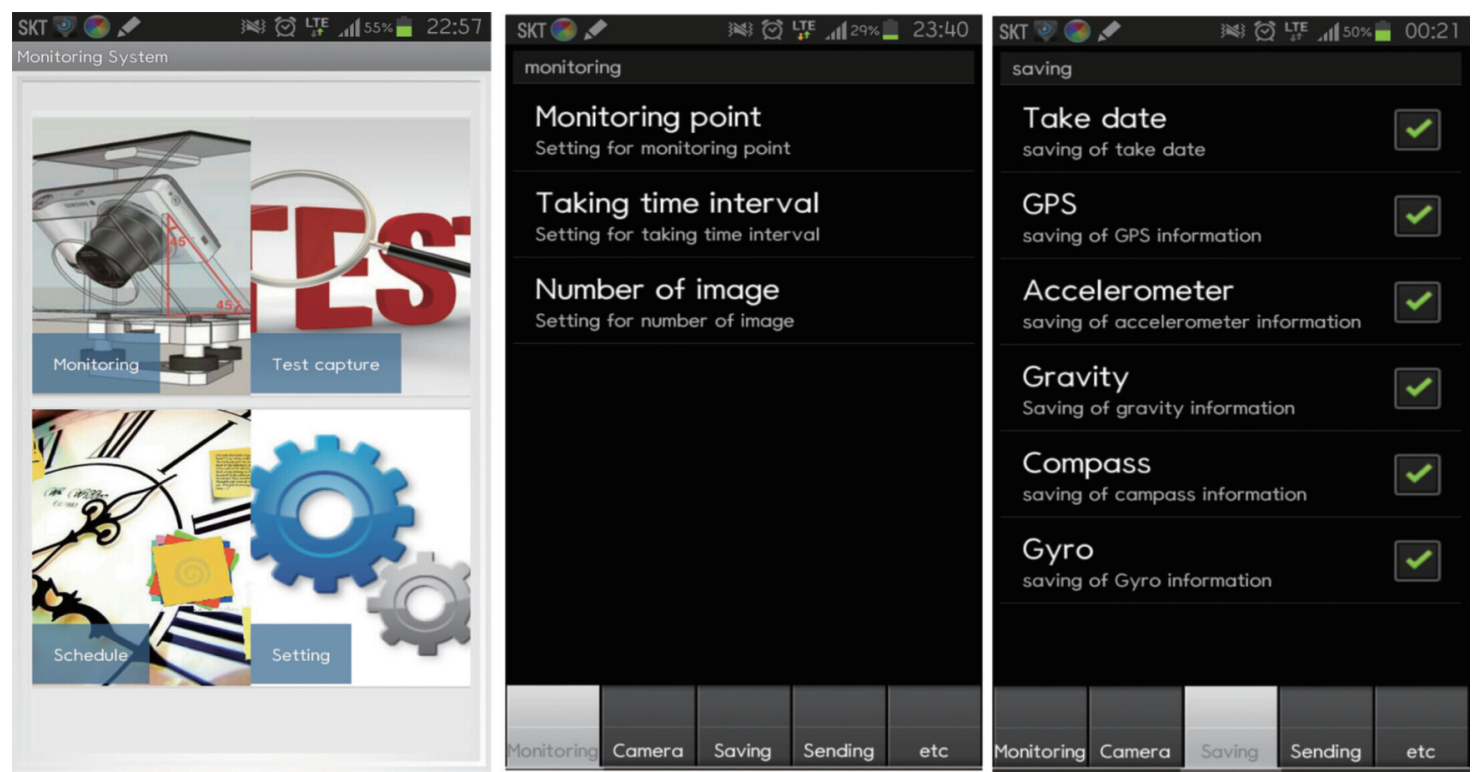

Fig. 3. Android application to control monitoring system.
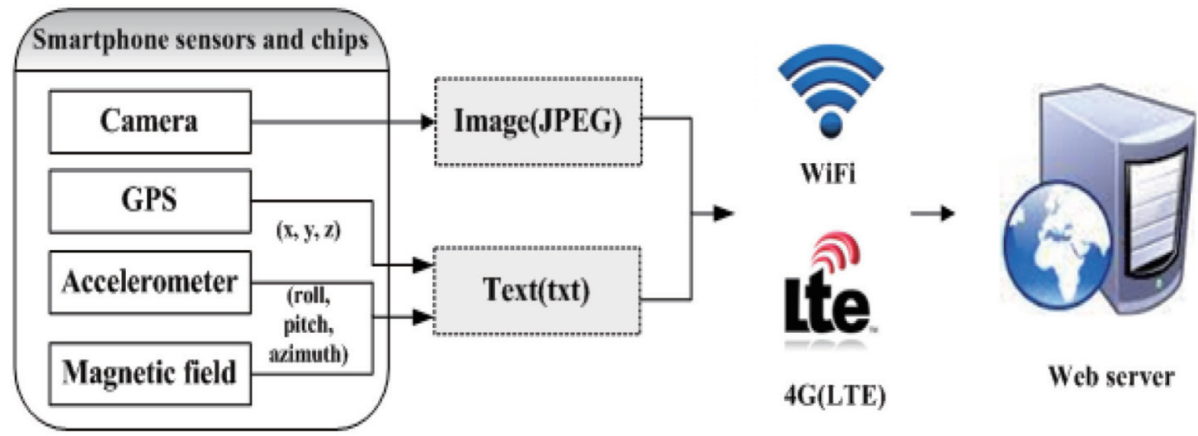

Fig. 4. Networks of the monitoring system transferring the camera sensor data.

The geo-referencing of images was performed by calculating the EO parameters of the camera with android-operated sensors using metadata (Chang, 2009). Inverse geo-referencing was obtained, not by the GCPs, but instead by the camera sensors (providing $\mathrm{x}, \mathrm{y}, \mathrm{z}, \omega, \phi$, and $\kappa$ data). Therefore, the inverse triangulation $\mathrm{EO}$ parameters were provided by the smartphone sensors (Fig. 4).

\section{2) Camera calibration}

Camera calibration was used to determine the IO parameters (e.g., FL, PP location, radian and tangential lens distortion) (Wolf, 2000) and EO parameters (Ricolfe-Viala, 2011). The SC may produce incorrect
ICs, due to lens distortion caused by the non-metric camera (Goshtasby, 1989); this is the main reason for 3-D positioning problems and shape distortion during orthorectification.

Camera calibration and the IO parameters were calculated using the PhotoModeler software (ver. 6, Eos System Inc.). Calibration was accomplished using two methods based on the calibration target sheet. STS calibration, the older calibration method of the two, uses a $1 \times 1-\mathrm{m}$ target sheet with a known pattern, produced specifically for calibration purposes (Fig. 4 (a)). MTS calibration (based on PhotoModeler, ver. 6) is a method that uses several sheets having ringed 

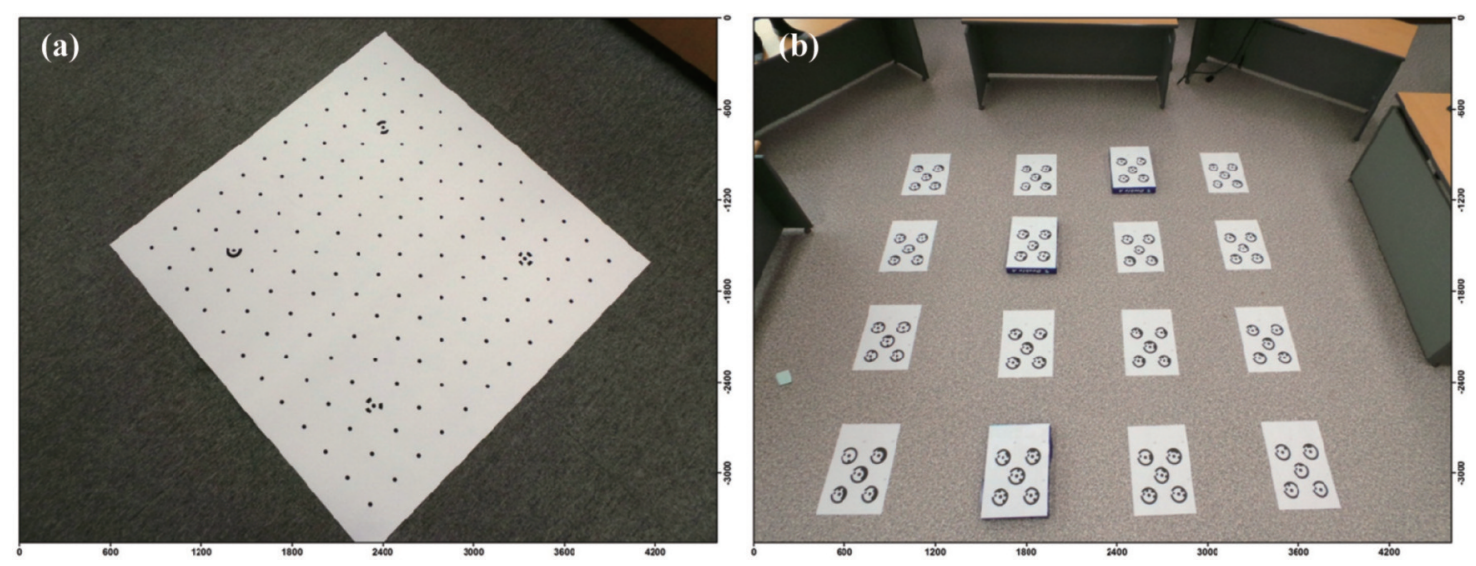

Fig. 5. Calibration sheet: (a) single-target and (b) multi-target for calibrations.

automatically detected (RAD) coded targets (Fig. 4(b)).

Taking into account the FL and CCD size/resolution of the camera, 16 A3-size sheets of RAD-coded targets were obtained (inner target diameter: $12.6 \mathrm{~mm}$; height: $1.8 \mathrm{~m}$; width: $1.8 \mathrm{~m}$ ), and different heights. For MTS and STS, a total of 16 sheets of images were acquired by each method, with the 16 sheets corresponding to the horizontal and vertical directions, from eight different camera directions.

The radial distortion difference ( $\mathrm{dr}$ ) in PhotoModeler expressed as

$$
d r=\mathrm{k}_{1} \cdot \mathrm{r}^{2}+\mathrm{k}_{2} \cdot \mathrm{r}^{4}+\mathrm{k}_{3} \cdot \mathrm{r}^{6}
$$$$
\text { where } \mathrm{r}^{\mathrm{i}}=\mathrm{x}^{\mathrm{i}}+\mathrm{y}^{\mathrm{i}}, \mathrm{i}=2,4 \text {, and } 6 \text {. }
$$

Tangential distortion (P1 and P2) also exists in lens distortion; however, it is insignificant and can thus be ignored (Fraser, 1997; Wolf, 2000). Therefore, the lens distortion consists of only the radial distortion component, as given in Eq. (1).

\section{3) Field experiments}

This study was carried out at the campus of Pukyung National University, located in Busan, Korea. This study acquired camera images from two locations, where the EO parameters were obtained by ground surveys. The DSM generated from stereo images was validated by comparison with the DSM created from the LiDAR data. To acquire point cloud data without

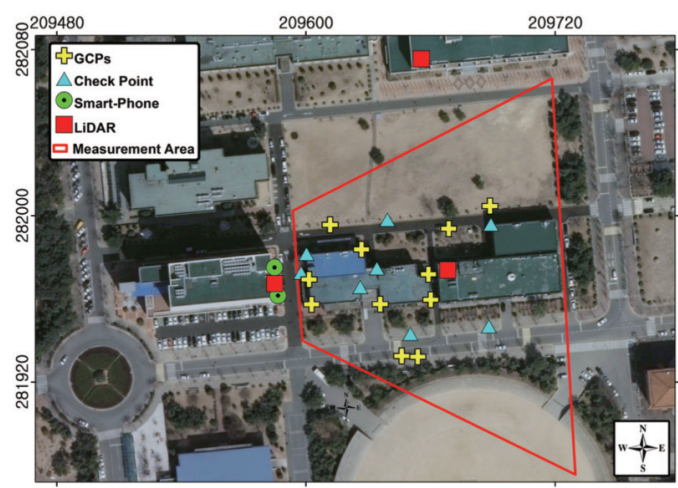

Fig. 6. The field test site distribution.

observation shadow zones, terrestrial LiDAR data from three locations was geo-referenced. The distribution of the GCPs and checkpoints is shown in Fig. 6.

The laboratory experiment zone was the Pukyung National University campus, which consisted of buildings, roads, trees, and grass. A terrestrial photo was taken by the Topcon 3-D Laser Scanner GLS1000; the photos merged into point clouds for terrestrial LiDAR, as shown in Fig. 7. The LiDAR point cloud data overlap on the terrestrial photo is shown in Fig. 6(b).

This study observed GCP coordinates from 30 locations using the SOKKIA CX-105 total station and Topcon GNSS receiver (GRX 1-U) GPS receiver. The DSM was generated using ERDAS Leica Photogrammetric Suites (LPS) (ver. 9.2), along with camera calibration parameters, IO and EO parameters, 

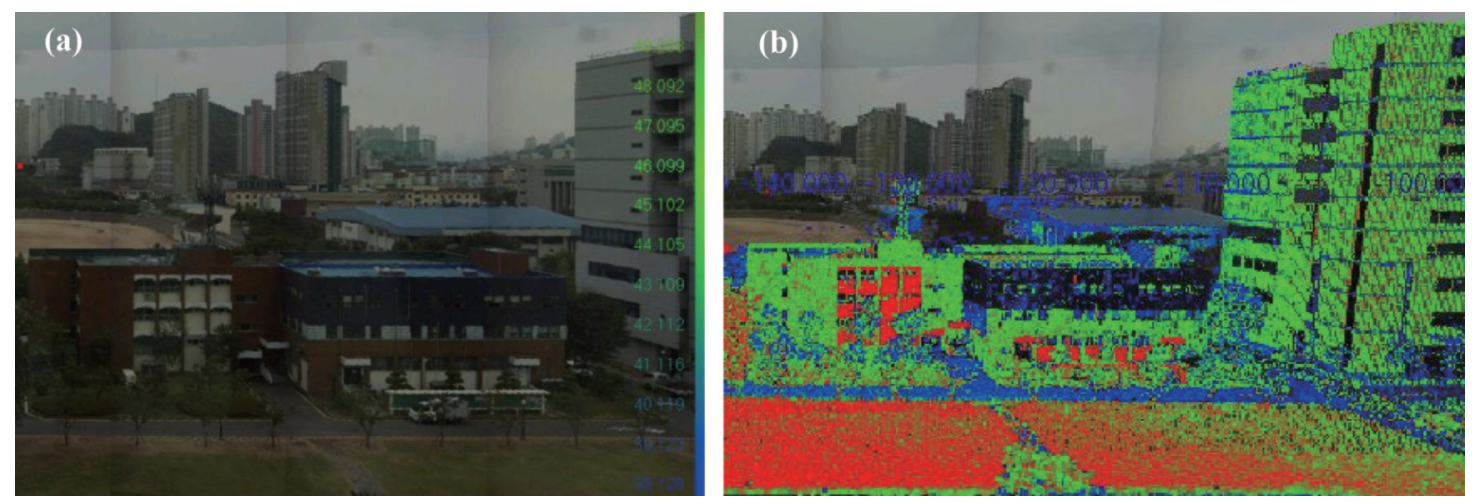

Fig. 7. Test field images: (a) mosaic of the terrestrial photos, (b) Image with LiDAR point cloud data.
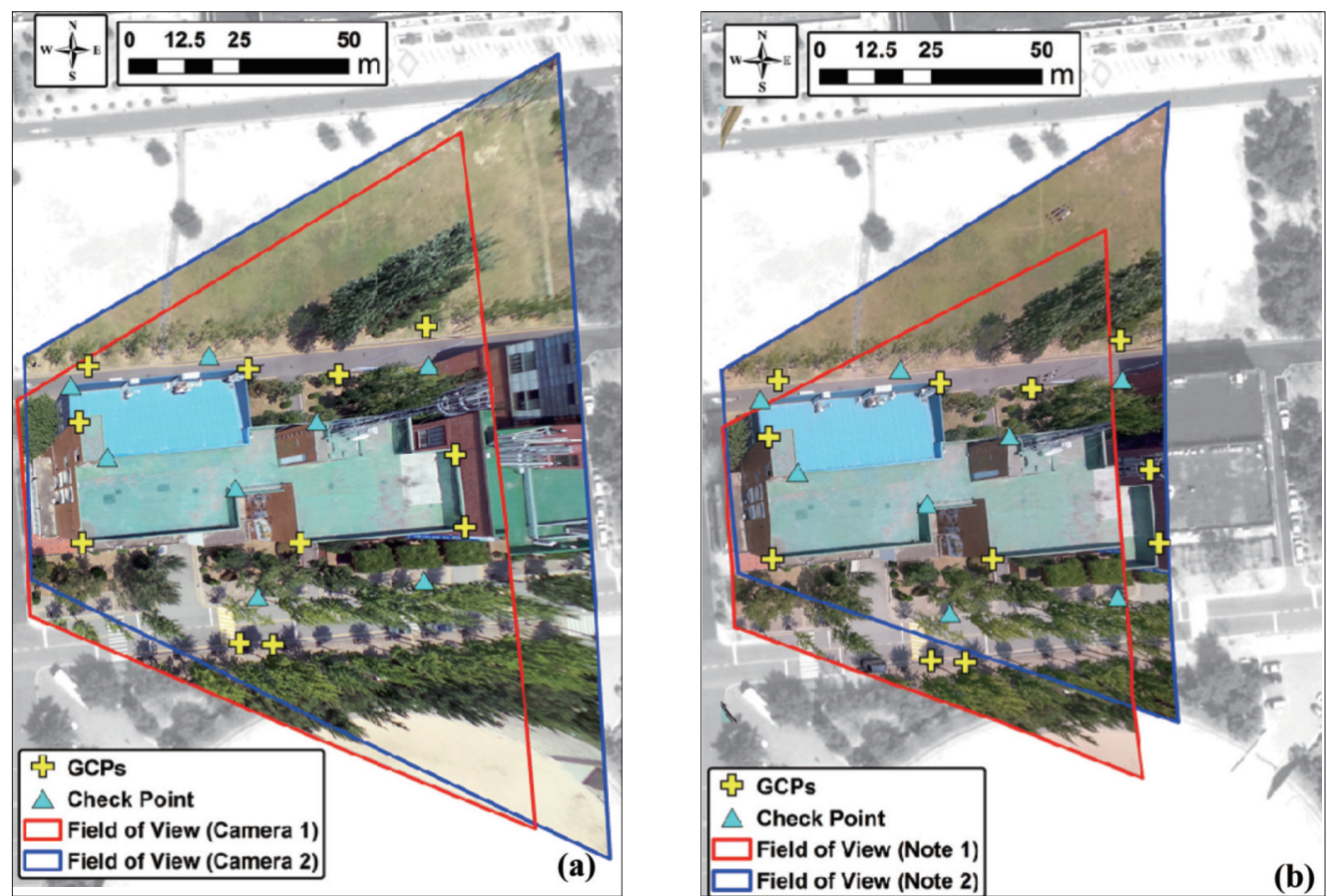

Fig. 8. Plot of GCPs, check points, measurement area, and projection outline of each camera : (a) SC and (b) SP.

and the GCP coordinates (ERDAS, 2011). The GCPs, check point distribution, and stereo model are shown in Fig. 8.

\section{Results and Discussion}

\section{1) Camera Calibration \& IO Parameter}

Calibrations and IO orientation methods, such as Single-Target Sheet (STS) and Multi-Target Sheet
(MTS) calibration, calculated using the perspective geometric model with bundle adjustment (Brown, 1971), can be used to correct for irregular lens distortion found on non-metric cameras. This study compared these calibration methods for two sets of SCs and SPs (SC: type 1, SC: type 2, SP: type 1, and SC: type 2). For both SC types, the FL was similar (or slightly longer) for each calibration method; whereas the FL varied by up to $0.02 \mathrm{~mm}$ depending on the calibration method used for the SPs. The $\mathrm{x}$ and $\mathrm{y}$ values of the PP coordinates, denoted PPx and PPy respectively, were 
Table 2. The results of Camera IO

\begin{tabular}{|c|c|c|c|c|c|c|c|c|}
\hline \multirow[b]{3}{*}{ Calibration target type } & \multicolumn{4}{|c|}{$\mathrm{SC}$} & \multicolumn{4}{|c|}{ SP } \\
\hline & \multicolumn{2}{|c|}{ Type 1} & \multicolumn{2}{|c|}{ Type 2} & \multicolumn{2}{|c|}{ Type 1} & \multicolumn{2}{|c|}{ Type 2} \\
\hline & MTS & STS & MTS & STS & MTS & STS & MTS & STS \\
\hline F.L. (mm) & 4.486 & 4.486 & 4.510 & 4.500 & 3.683 & 3.666 & 3.673 & 3.663 \\
\hline P.P.x(mm) & 0.040 & 0.048 & 0.012 & 0.002 & 0.005 & 0.003 & 0.023 & 0.013 \\
\hline P.P.y(mm) & 0.043 & 0.041 & 0.118 & 0.118 & -0.001 & -0.009 & 0.016 & 0.009 \\
\hline Pixel size $(X, Y)(\mu \mathrm{m})$ & 1.400 & 1.400 & 1.400 & 1.400 & 1.400 & 1.400 & 1.400 & 1.400 \\
\hline Image coordinate RMSE (pixel) & 0.458 & 0.494 & 0.530 & 0.534 & 0.632 & 0.515 & 0.549 & 0.663 \\
\hline $\begin{array}{c}\text { Adjusted target coordinate } \\
\text { RMSE }(\mathrm{mm})\end{array}$ & 0.058 & 0.146 & 0.067 & 0.166 & 0.115 & 0.258 & 0.101 & 0.336 \\
\hline K1 (Lens Radial Distortion) & $4.58 \mathrm{e}^{-04}$ & $4.21 \mathrm{e}^{-04}$ & $1.27 \mathrm{e}^{-03}$ & $1.22 \mathrm{e}^{-03}$ & $-9.05 \mathrm{e}^{-03}$ & $-8.97 \mathrm{e}^{-03}$ & $-9.41 \mathrm{e}^{-03}$ & $-8.99 \mathrm{e}^{-03}$ \\
\hline K2 & $-1.08 \mathrm{e}^{-05}$ & $-3.92 \mathrm{e}^{-06}$ & $-1.55 \mathrm{e}^{-05}$ & $-5.56 \mathrm{e}^{-06}$ & $1.17 \mathrm{e}^{-03}$ & $1.12 \mathrm{e}^{-03}$ & $1.20 \mathrm{e}^{-03}$ & $1.06 \mathrm{e}^{-03}$ \\
\hline K3 & 0.000 & 0.000 & 0.000 & 0.000 & 0.000 & 0.000 & 0.000 & 0.000 \\
\hline P1 (Lens Tangential Distortion) & $5.66 \mathrm{e}^{-04}$ & $5.66 \mathrm{e}^{-04}$ & $2.76 \mathrm{e}^{-04}$ & $1.04 \mathrm{e}^{-04}$ & $8.86 \mathrm{e}^{-05}$ & $3.82 \mathrm{e}^{-04}$ & $3.36 \mathrm{e}^{-04}$ & 0.00 \\
\hline $\mathrm{P} 2$ & $-5.32 \mathrm{e}^{-04}$ & $-5.32 \mathrm{e}^{-04}$ & $-1.64 \mathrm{e}^{-03}$ & $-1.64 \mathrm{e}^{-03}$ & 0.00 & $1.21 \mathrm{e}^{-04}$ & $1.10 \mathrm{e}^{-03}$ & 0.00 \\
\hline RMSE & 0.458 & 0.458 & 0.530 & 0.540 & 0.632 & 0.515 & 0.549 & 0.663 \\
\hline Max residual & 1.851 & 1.851 & 1.740 & 3.202 & 4.119 & 2.774 & 3.545 & 4.061 \\
\hline
\end{tabular}

* IO: interior orientation, F.L.: Focal length, P.P.: Principal point

similar for SC type 1; the PPx value varied for SC type 2 depending on the calibration method, and were larger than the PPy values. The SP types exhibited the same PPx and PPy values for both calibration methods. Both SC types, and SP type 1 showed almost identical PP values and offset directions, regardless of the differences in the calibration sheets. However, for SP type 2, the PP values were affected by the calibration sheet. For SP type 2, the PP values calculated by the MTS calibration were approximately 0.5 -fold higher than the STS calibration.

The Root Means Square Error (RMSE) of image coordinates for SC type 1 was $<0.5$ pixels, whereas it was slightly larger than 0.5 pixels for SC type 2 . For SP type1, the RMSE of image coordinates is always larger than 0.5 pixels and varies based on the calibration method. For the RMSE of adjusted target coordinates, the MTS calibration produced more than two times accurate than the STS method. As evidence by the lower RMSE values, the lens distortion of SC caused by the perspective projection was smaller than that of $\mathrm{SP}$, because multi-lens construction SC is more stable lens than single-lens construction SP (Table 2).
The distortion at the center of the lens is not calibration-method dependent, whereas marked differences were evident around the edge of the image. The radial distortion for SC type 1 shows a maximum value at the edge, with an increase in monotone.

Depending on the calibration target sheet, the radial distortion differences increased with distance from the perspective center to a maximum of $1.172 \mu \mathrm{m}$ at the edge of the image. The radial distortion for SC type 2 was similar to that for SC type 1; a maximum difference in radial distortion of $1.844 \mu \mathrm{m}$ at the edge was observed for the calibration target sheets. Radial distortion for SP type 1 exhibited a maximum value 2 $\mathrm{mm}$ away from the perspective center. The differences in radial distortion for different calibration target sheets showed similar trends to the $\mathrm{SC}$, indicating a maximum difference of $2.535 \mu \mathrm{m}$ at the edge. SP type 2 exhibited a similar pattern and values as SP type 1 . The difference in the radial distortion, depending on the calibration target sheet, showed a maximum of $5.393 \mu \mathrm{m}$ at the edge. The radial distortion with MTS calibration was slightly smaller than that with STS calibration.

The FL and PPx and PPy coordinates were 
(a)

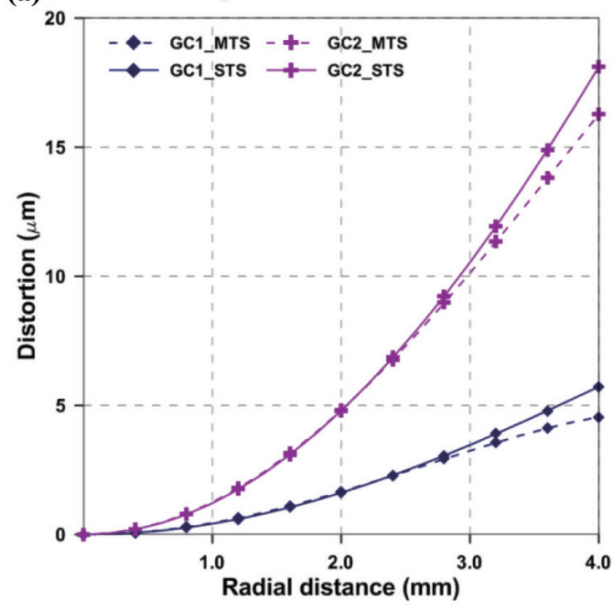

(b) Galaxy note2 lens distorion

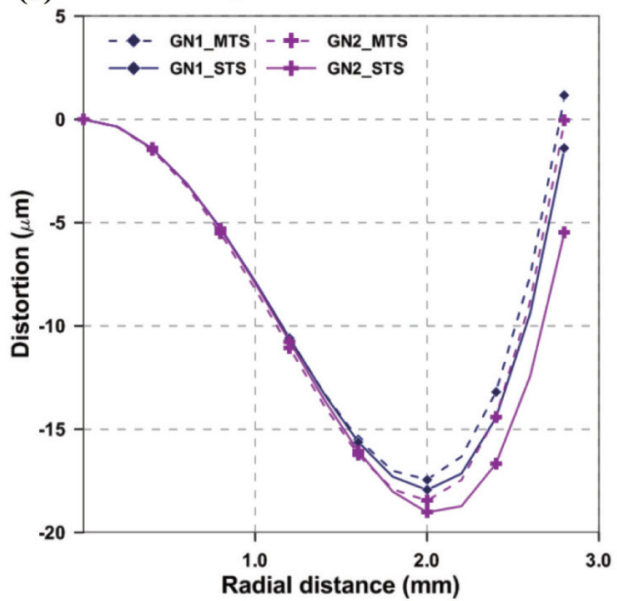

Fig. 9. Comparison of the radial distortion curve between STS and MTS lens distortion calibration.

Table 3. Comparison with the results of other recent research about non-metric Camera

\begin{tabular}{|c|c|c|c|c|c|c|c|}
\hline \multirow{2}{*}{ Researcher } & \multirow{2}{*}{ Camera } & \multirow{2}{*}{ Method } & \multicolumn{2}{|c|}{ F.L. (mm) } & \multirow{2}{*}{$\begin{array}{l}\text { P.P.x } \\
(\mathrm{mm})\end{array}$} & \multirow{2}{*}{$\begin{array}{l}\text { P.P.y } \\
(\mathrm{mm})\end{array}$} & \multirow{2}{*}{$\begin{array}{l}\text { RMSE } \\
\text { (pixel) }\end{array}$} \\
\hline & & & By company & Calculated & & & \\
\hline \multirow{8}{*}{ This reseach } & \multirow{2}{*}{ SC type1 } & MTS & $4.1 \sim 86.1$ & 4.49 & 0.040 & 0.043 & 0.458 \\
\hline & & STS & $4.1 \sim 86.1$ & 4.49 & 0.048 & 0.041 & 0.494 \\
\hline & \multirow{2}{*}{ SC type 2} & MTS & $4.1 \sim 86.1$ & 4.51 & 0.012 & 0.118 & 0.53 \\
\hline & & STS & $4.1 \sim 86.1$ & 4.5 & 0.002 & 0.118 & 0.534 \\
\hline & \multirow{2}{*}{ SP type1 } & MTS & 3.7 & 3.68 & 0.005 & 0.003 & 0.632 \\
\hline & & STS & 3.7 & 3.67 & -0.001 & -0.009 & 0.515 \\
\hline & \multirow{2}{*}{ SP type 2} & MTS & 3.7 & 3.67 & 0.023 & 0.016 & 0.549 \\
\hline & & STS & 3.7 & 3.66 & 0.013 & 0.009 & 0.663 \\
\hline \multirow{2}{*}{ Fraser } & \multirow{2}{*}{ Nikon D200 } & Static & 60 & 59.21 & -0.32 & 0.47 & 0.11 \\
\hline & & Kinematic & 60 & 59.19 & -0.25 & 0.26 & 0.13 \\
\hline Sužiedelytė-Visockienè & Cannon EOS 1D Mark-II & & - & 19.65 & -0.11 & 0.06 & 0.43 \\
\hline Gneeniss & Vexcel UltraCamX & & 100.5 & 100.49 & 0.01 & -0.01 & $3.5(*)$ \\
\hline Perez & Pentaxs Optio A40 & & $12 \sim 37$ & 8.19 & 0.01 & 0.14 & $10.05(*)$ \\
\hline
\end{tabular}

(*): The RMSE of check points (unit: $\mathrm{cm}$ )

compared to the research results for the non-metric camera calibrations (Table 4). The FL was divided into nominal FL (specified by the manufacturer) and calibrated FL (determined from camera calibration). Nominal FL and calibrated FL vary depending on the distance between the target and the camera lens. The study by Fraser (2012) showed $0.02 \mathrm{~mm}$ of difference in the static and kinematic FLs for non-metric cameras. The PPx and PPy values were 1.5- to 2-fold larger, and the RMSE of the ICs was 0.02 pixels smaller in the static environment, compared with kinematic environments. The RMSE of the ICs of SužiedelytèVisockienè (2012) was 0.43 pixels, indicating a similar value to the $\mathrm{SC}$ in this study. Owing to its single-lens construction, the RMSE of ICs for SP has a larger value than multi-lens construction SCs, but can be reduced to $\sim 0.5$ pixels with lens distortion correction. Although large differences for the IO parameters were not 
observed, MTS calibration has been shown to be more reliable than STS calibration (PhotoModeler Tutorial for radial distortion and adjusted target coordinate RMSE).

\section{2) Field experiments for $3-D(x, y, z)$}

For a constructed combined traverse with the Sokkia Total station CX-105, the errors of disclosure for the $\mathrm{X}, \mathrm{Y}$, and Z directions of the GCPs were ?0.018, 0.027, and $? 0.001 \mathrm{~m}$ respectively, with the error of disclosure of combined traverse of $0.019 \mathrm{~m}$. The ratio of error closure of combined traverse was calculated to be 1/8763; the measured coordinates can be used for the GCPs. This study calculated the RMSE value for the ICs using 12 to 15 GCPs among 30 measured GCPs (with image verification). Additionally, the geometric strength of the stereo image was calculated from the RMSE value of the ICs, using the remaining GCPs as checkpoints. This paper calculated the geometric strength by changing the calibration settings and analyzing the effects for three cases: MTS calibration, STS calibration, and no lens calibration.

Experiments are divided into six configurations. Each configuration consists of one of three-case calibrations and one of two-type aerial triangulations (aerial triangulation by GCP and inverse aerial triangulation by Camera EO) (Figure 10). For SC, the RMSE value for the image coordinates ICs calculated by aerial triangulation, was shown to be $<0.5$ pixels for both MTS and STS calibrations. For inverse aerial triangulation measurements, the RMSE for the y-axis direction was less accurate, compared with the $\mathrm{x}$-axis direction, regardless of the calibration method. Although the $\mathrm{x}$-axis direction showed similar RMSE values for each calibration method, the RMSE for the $\mathrm{y}$-axis direction was larger by 2.0 pixels for the no-lens calibration method. The RMSE for SPs exhibited a similar to slightly increasing pattern, compared with SCs with no lens calibration. This indicated that the single-lens construction SPs produced more distortion than the SCs. The RMSE for image coordinates calculated by inverse aerial triangulation showed a 2?3times increase. Thus, MTS calibration was shown to be the most precise calibration method, regardless of the camera type; additionally, geo-referencing for SP images with single-lens construction is critical for camera calibration, similar to the results of Kim et al. (2013b).

Based on various viewpoints, this study conducted analysis for each stereo pair to compare the 3-D positional accuracy for the stereo models. This was done by photographing images at known intervals, and calculating the stereo models from coordinates from check points for the $\mathrm{x}-, \mathrm{y}-$, and z-axes (Fig. 11). Twenty check points for the SCs and 15 check points for the $\mathrm{SPs}$ were used. For the SCs, all values were $<0.474 \mathrm{~m}$, except for one RMSE value corresponding to the $\mathrm{x}$-axis direction of the stereo model. The 3-D positional
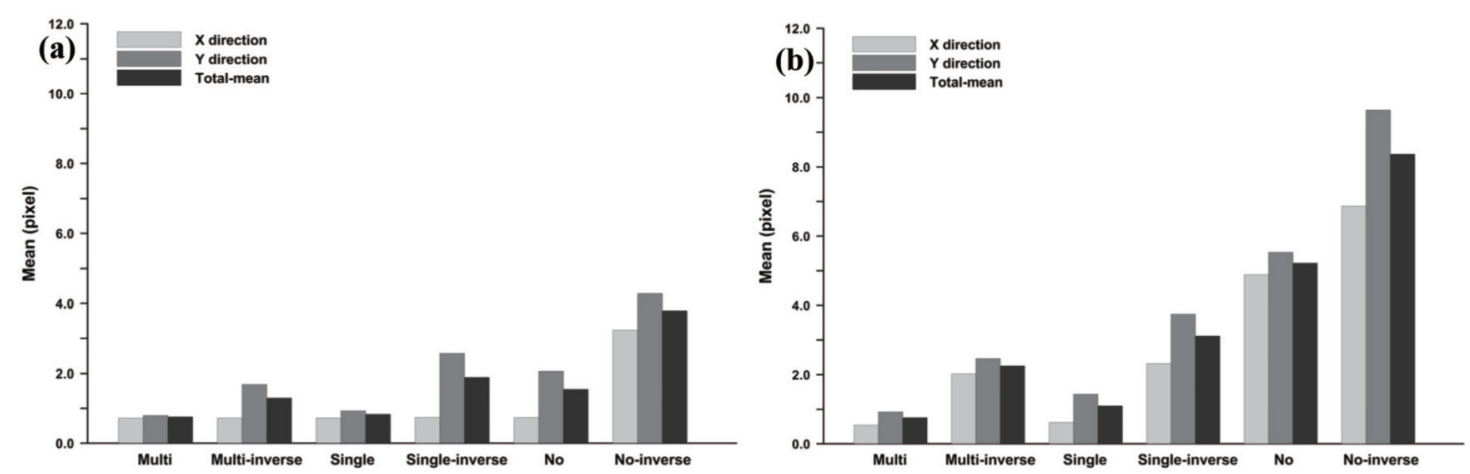

Fig. 10. Plot of image coordinates RMSE in pixels of each Calibration Method : (a) SC and (b) SP. 

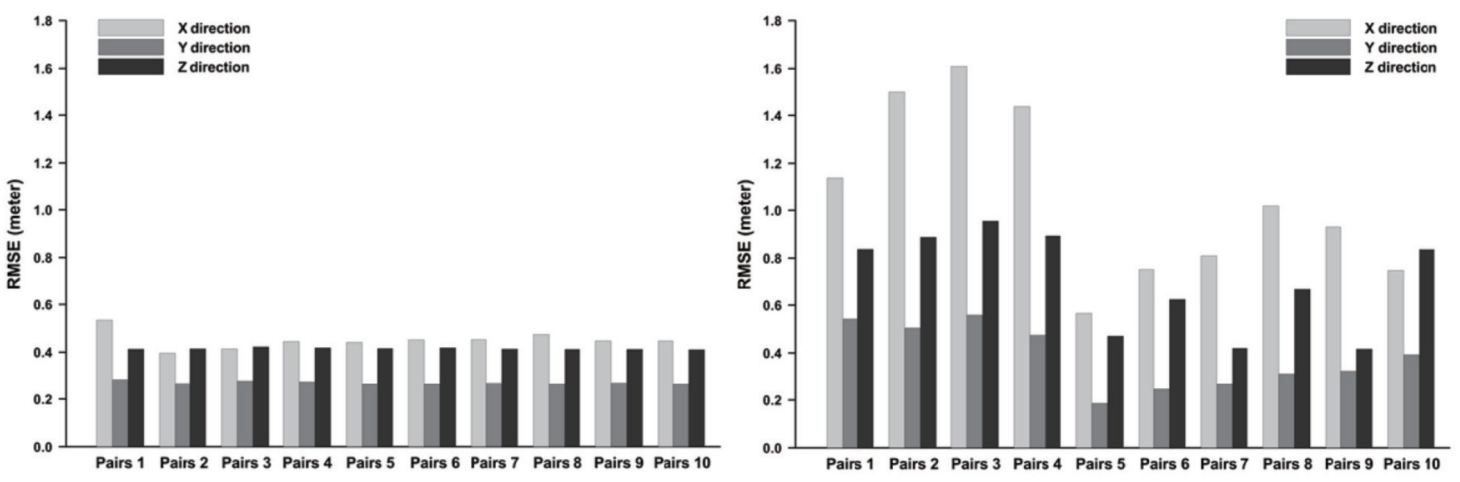

Fig. 11. Check points' RMSEs stereo image pairs captured in different time: (a) SC and (b) SP.

accuracy analysis for the stereo model did not depend on the time of picture acquisition. The RMSE of the coordinates of the checkpoints for the $\mathrm{x}$ - and $\mathrm{z}$-axes were similar. The RMSE value for the $y$-axis was $<0.29 \mathrm{~m}$, and was the most accurate of all axes directions for both SPs and SCs. The RMSE values for all axes directions were larger for the SPs, compared with the SCs and with the x-axis direction. Especially, it increases up to $1.61 \mathrm{~m}$ from $\mathrm{X}$-axis and variations in coordinates of $\mathrm{X}$-axis in checkpoints are higher and inaccurate. Therefore, RMSE value for checkpoints' coordinates depending on stereo pairs of SC is same. However, For SP, as its RMSE value is varying depending on the time of capturing image, the result of geo-referencing is different depending on image capture time.

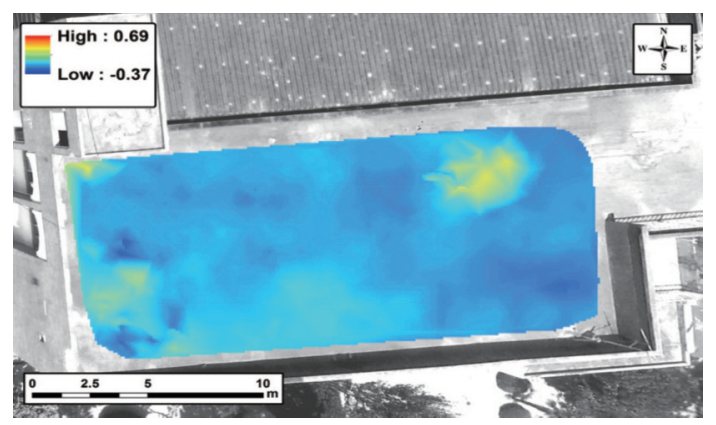

(a)
The 3-D positional accuracy for non-metric cameras has a 0.2-pixel RMSE when high-resolution calibration is conducted (Rieke-Zapp, 2005; D'Amelio, 2009). Several studies have shown $<0.3-0.5$ pixels RMSE in their 3-D positional accuracy (Akca, 2009; Chandler, 2005; Hinz, 2009).

DSMs were created from images using the above six configurations (Figure 12). The calculated differences from DSM created by LiDAR data (DSM-1) are shown. The DSM generated with SCs experienced similar changes in the DSM-1, depending on camera calibration. Unlike SC, the height of DSM generated with SP was greater at the bottom left side compared with that shown in the DSM-1, whereas it decreased gradually as it moved to the right. Little change in the color during STS calibration indicated little difference

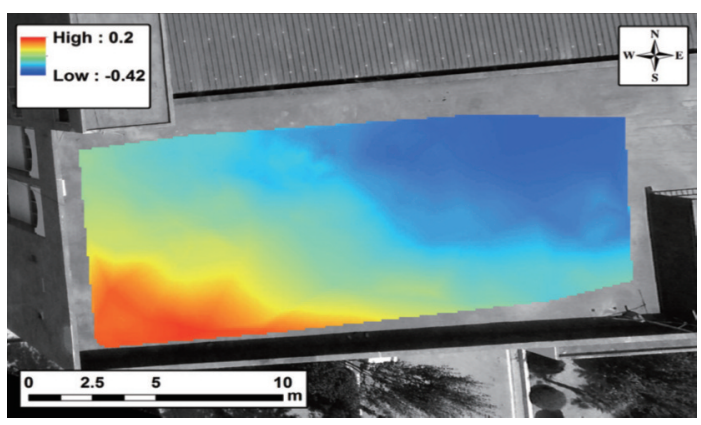

(b)

Fig. 12. Comparison of a digital surface model generated by LiDAR : DSM of SC with MTS calibration, (b) DSM of SP with MTS, (c) DSM of SC with STS calibration, (d) DSM of SP with STS calibration, (e) DSM of SC without lens calibration, and (f) DSM of $\mathrm{SP}$ without lens calibration (unit: $\mathrm{m}$ ). 


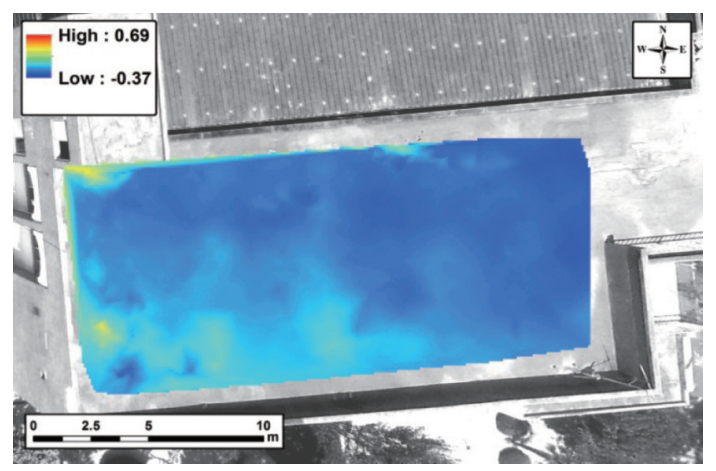

(c)

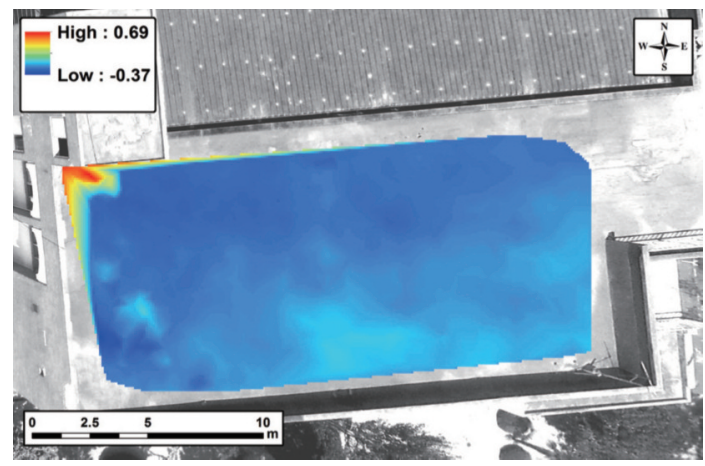

(e)

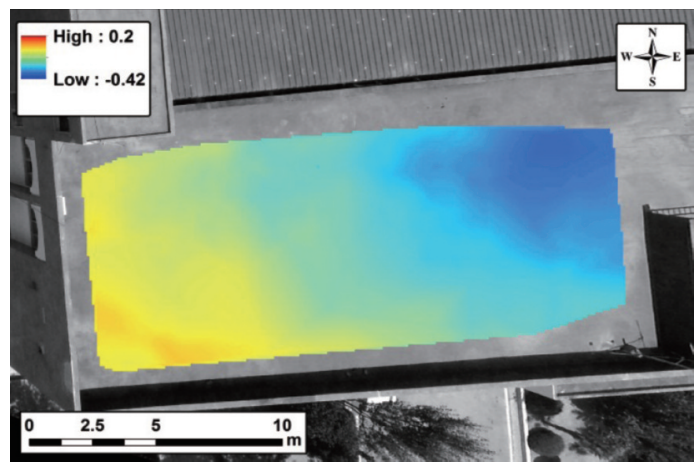

(d)

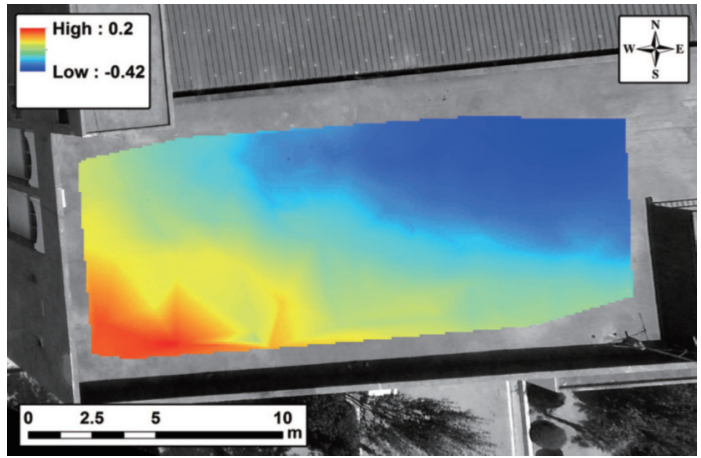

(f)

Fig. 12. Continued.

in the DSM-1, compared with other calibration methods. For SP, the distortion in the ground height value varied depending on the image location.

This study carried out statistical analysis of the difference between the DSM and DSM-1 created by each calibration method (Table 4). The differences between DSM-1 and DSM generated by the camera calibration had an average value of $0.180 \mathrm{~m}$. The standard deviation was less than $0.076 \mathrm{~m}$ for SCs and $0.138 \mathrm{~m}$ for SPs when there was camera calibration.

The average value and standard deviation between DSM increased in the absence of camera calibration. The accuracy and precision for DSM-MTS calibration was highest for the SCs; whereas, STS calibration provided the best results for SPs. Although the accuracy of the 3-D location was more or less different, depending on the camera type and calibration target sheet used, overall, camera calibration enhanced the accuracy of the 3-D locations. The standard deviation for the DSM shows that this method exceeds

Table 4. Differences of between LiDAR DSM and DSM generated by LPS (unit: $\mathrm{m}$ )

\begin{tabular}{c|c|c|c|c|c}
\hline \hline \multicolumn{2}{c|}{ Camera } & Mean & Standard deviation & Min & Max \\
\hline \multirow{3}{*}{ SC } & MTS & -0.106 & 0.065 & -0.256 & 0.287 \\
\cline { 2 - 6 } & STS & -0.161 & 0.076 & -0.284 & 0.352 \\
\cline { 2 - 6 } & No Cal. & -0.166 & 0.091 & -0.364 & 0.689 \\
\hline \multirow{3}{*}{ SP } & MTS & -0.18 & 0.138 & -0.354 & 0.181 \\
\cline { 2 - 6 } & STS & -0.164 & 0.102 & -0.341 & 0.033 \\
\cline { 2 - 6 } & No Cal. & -0.182 & 0.148 & -0.415 & 0.196 \\
\hline \hline
\end{tabular}


Table 5. The positional accuracy of Camera positions along each axis and their RMSE. (unit : $\mathrm{m}$ )

\begin{tabular}{|c|c|c|c|c|c|}
\hline Camera & Type & $\mathrm{dx}$ & dy & $\mathrm{dz}$ & Total RMSE \\
\hline \multirow{9}{*}{$\mathrm{SC}$} & MTS & 0.119 & 0.08 & -0.063 & 0.223 \\
\hline & Type 1 & 0.077 & -0.082 & -0.055 & 0.125 \\
\hline & Type 2 & 0.321 & 0.242 & -0.07 & 0.408 \\
\hline & STS & 0.24 & 0.094 & -0.091 & 0.274 \\
\hline & Type 1 & 0.076 & -0.104 & -0.063 & 0.143 \\
\hline & Type 2 & 0.405 & 0.292 & -0.119 & 0.513 \\
\hline & No Cal. & 0.139 & 0.047 & -0.031 & 0.15 \\
\hline & Type 1 & 0.233 & 0.193 & -0.026 & 0.303 \\
\hline & Type 2 & 0.045 & -0.099 & -0.037 & 0.115 \\
\hline \multirow{9}{*}{ SP } & MTS & 0.667 & -0.014 & -0.653 & 0.933 \\
\hline & Type 1 & 0.419 & 0.138 & -0.506 & 0.672 \\
\hline & Type 2 & 0.915 & -0.166 & -0.799 & 1.226 \\
\hline & STS & 0.541 & 0.03 & -0.571 & 0.787 \\
\hline & Type 1 & 0.571 & 0.145 & -0.643 & 0.872 \\
\hline & Type 2 & 0.511 & -0.086 & -0.499 & 0.719 \\
\hline & No Cal. & 0.979 & -0.002 & -0.899 & 1.329 \\
\hline & Type 1 & 0.75 & 0.136 & -0.793 & 1.1 \\
\hline & Type 2 & 1.209 & -0.139 & -1.005 & 1.578 \\
\hline \multicolumn{2}{|c|}{ Average } & 0.461 & 0.039 & -0.385 & 0.616 \\
\hline
\end{tabular}

Chandler's (2005) results.

This study calculated the perspective center coordinates of the GCP using the IO and EO parameters of the camera and perspective center coordinates to verify the accuracy of the monitoring system. By setting the standard deviation of the GCP positional accuracy to $0.1 \mathrm{~m}$, the accuracy in the locations of the two cameras were determined from the differences in their $\mathrm{x}, \mathrm{y}$, and $\mathrm{z}$ axes and RMSE values (Table 5). To analyze the effects on the calculation of the camera location by the calibration environment, the RMSE of the camera location was calculated by changing the calibration setting. Although the MTS and STS calibration results were not much different when the calibration method of the SC was used, the MTS calibration provided slightly better accuracy. However, when camera lens calibration was not performed, the accuracy of the camera location was better than the camera calibration space-resectioning procedure. SPs showed a different trend, compared with the SCs.
Based on the accuracy of the installed camera location, given that the accuracy of the SP was the RMSE value, the results were in the following order of superiority: STS calibration, MTS calibration, and no lens calibration.

\section{Conclusions}

A 4D ubiquitous monitoring system using Android 4.1.2 was investigated, based on trials with a 3-D monitoring system. This study also carried out two camera calibration methods to create a camera model by the removal of lens distortion. The IO parameter, FL, as well as the radial distortion coefficients showed more precise values by MTS calibration than by STS calibration. Coordinate movement for PP in SCs was larger than that for SPs. The larger movement of the perspective center was attributed to variation in the PP value resulting from lens tube movement of the 
motorized zoom digital camera model. This study also confirmed that STS calibration provided the highest accuracy of camera location for SPs. The calculated camera location for SCs varied over time, due to changes in the IO parameters of the SCs with changes in the zoom lens.

RMSE for check points calculated by bundle adjustment showed the best result for MTS calibration in SC and for STS calibration in SP. This study compared geo-referenced data of LiDAR with created DSM to validate the accuracy of DSM created by stereo. The RMSE was ?0.106 m for SCs, which was lower that for SPs $(? 0.180 \mathrm{~m})$. This was attributed to the greater precision provided by the lens distortion compensation of the SC, compared with the SP with single lens construction and lens tube instability. This study also compared the location of moving targets calculated by the stereo model with the location provided by an attached GPS to calculate and validate the accuracy of the location of the ground coordinates of moving targets. When camera calibration was performed to measure the difference in the two coordinates for the moving targets, its value was $<6.5$ $\mathrm{cm}$. This means that even SPs, equipped with nonmetric cameras, can be used for monitoring purposes.

In the future, as IT advances, specifications of mobile devices become enhanced, algorithms are available to minimize memory usage, and if real-time processing becomes available without the need to send data to a server under mobile environments, then simple monitoring systems such as that proposed herein will become feasible.

\section{Acknowledgment}

This research was financially supported by the Ministry of Education (MOE) and National Research Foundation of Korea(NRF) through the Human Resource Training Project for Regional Innovation No.
2013 H1B8A2027455.

\section{References}

Akca, D., and A. Gruen, 2009. Comparative geometric and radiometric evaluation of mobile phone and still video cameras, Photogramm. Rec., 24: 217-245.

Botan, C. and M. Vorvoreanu, 2005. What do employees think about electronic surveillance at work?, Electronic Monitoring in the Workplace: Controversies and Solutions, Idea Group Publishing.

Brown, D., 1971. Close-range Camera calibration, $P E \& R S, 37: 855-866$.

Cas, J., 2005. Privacy in pervasive computing environments - a contradiction in terms, IEEE Technology and Society Magazine, 24(1): 2433.

Chandler, J. H., J.G. Fryer, and A. Jack, 2005. Metric capabilities of low-cost digital cameras for close range surface measurement, Photogramm. Rec., 20: 12-26.

Chang, Y., C. Chen, and S. Zhou, 2009. Smart phone for mobile commerce, Computer Standards \& Interfaces, 31: 740-747.

Coroama, V., J. Bohn, and F. Mattern, 2004. Living in a smart environment - implications for the coming ubiquitous information society, IEEE international Conference on Systems, Man and Cybernetics, 6: 5633-5638.

D’Amelio, S., and M.L. Brutto, 2009. Close range photogrammetry for measurement of paintings surface deformations, International Archives of Photogrammetry, Remote Sensing and Spatial Information Sciences, 38(5/W1): 6. Erdas, Intergraph corporate website, 2011. < http:// www.erdas.com/>

Fleck, S. and W. Strasser, 2008. Smart Camera Based 
Monitoring System and Its Application to Assisted Living, Proc. of the IEEE, Vol.96 (10), pp. 1698-1714.

Fraser, C.S., 2012. Automatic Camera calibration in close-range photogrammetry, Photogrammetric Engineering \& Remote Sensing, 79(4): 381388.

Fraser, C.S., 1997. Digital camera self-calibration. ISPRS Journal of Photogrammetry and Remote Sensing, 52(4): 149-159.

Google, 2012. Android Developers websites. <http:// developer.android.com/develop/ index.html $>$ Android ver. 4.1.2.

Goshtasby, A., 1989. Correction of image deformation from lens distortion using Bezier patches, Computer vision, Graphics, and Image Processing, 47(3): 358-394.

Hinz, S., M. Stephani, L. Schiemann, and K. Zeller, 2009. An image engineering system for the inspection of transparent construction materials, ISPRS J. Photogramm. Remote Sens., 64: 297-307.

Joundi, R.A., J.S. Brittain, N. Jenkinson, A.L. Green, and T. Aziz, 2011. Rapid tremor frequency assessment with the iPhone accelerometer, Parkinsonism and Related Disorders, 17(4): 288-290.

Kim, J., S. Lee, H. Ahn, D. Seo, J. Lee, and C. Choi, 2013a. Accuracy evaluation of a smartphonebased technology for coastal monitoring, Measurement, 46(1): 233-248.

Kim, J., S. Lee, H. Ahn, D. Seo, S. Park, and C. Choi, 2013b. Feasibility of employing a smartphone as the payload in a photogrammetric UAV system, ISPRS Journal of Photogrammetry and Remote Sensing, 79: 1-18.

Lee S, J. Kim, C. Jin, S. Bae, and C. Choi, 2012a. Assessment of smartphone based technology for remote environmental monitoring and its development, Instrumentation Science \&
Technology, 40(6): 504-529.

Lee S, C. Jin, J. Kim, and C. Choi,2012b. Development of a smartphone application for environmental monitoring, Proc. of the ASPRS Annual Conference, Sacramento California, pp. 19-23.

Monserrat, O. and M. Crosetto, 2008. Deformation measurement using terrestrial laser scanning data and least squares 3D surface matching, ISPRS Journal of Photogrammetry and Remote Sensing, 63: 142-154.

Moran, S. and K. Nakata, 2010. Analyzing the Factors Affecting Users in Intelligent Pervasive Spaces, Intelligent Buildings International, 2(1): 57-71.

Pedersini, F., A. Sarti, and S. Tubaro, 1999. Accurate and simple geometric calibration of multicamera systems, Signal Process., 77: 309-334.

Ricolfe-Viala, C., and A. Sánchez-Salmerón, 2011. Using the camera pin-hole model restrictions to calibrate the lens distortion model, Opt. Laser Technol., 43: 996-1005.

Rieke-Zapp, D.H., and M.A. Nearing, 2005. Digital close range photogrammetry for measurement of soil erosion, Photogramm. Rec., 20: 69-87.

Singh, S., S. Puradkar, and Y. Lee, 2006. Ubiquitous computing: connecting pervasive computing through Semantic Web, Information Systems and E-Business Management, 4(4): 421-439.

Sužiedelytè-Visockienè, J., 2012. Photogrammetry requirements for digital Camera calibration applying Tcc and MatLab software, Geodesy and Cartography, 38(3): 106-110.

Tessens, L., M. Morbée, H. Aghajan, and W. Philips, 2014. Camera Selection for Tracking in Distributed Smart Camera Networks, ACM Transactions on Sensor Networks, 10(2): 23.

Wolf, P.R., and B.A. Dewitt, 2000. Elements of photogrammetry with Application in GIS, McGraw-Hill Book Company, New York. 